\title{
Progressive Obesity in Female Rats from Synergistic Interactions between Drugs and Whole Body Application of Weak, Physiologically Patterned Magnetic
} Fields

\author{
Linda S. St-Pierre, Michael A. Persinger \\ Behavioural Neuroscience Program, Laurentian University, Sudbury, Canada \\ Email: Ix stpierre@laurentian.ca, mpersinger@laurentian.ca
}

Received 23 April 2014; revised 4 June 2014; accepted 22 June 2014

Copyright (C) 2014 by authors and Scientific Research Publishing Inc.

This work is licensed under the Creative Commons Attribution International License (CC BY).

http://creativecommons.org/licenses/by/4.0/

c) (i) Open Access

\begin{abstract}
Prepubescent female rats treated with the atypical neuroleptic acepromazine but not ketamine, prazosin, or doxepin, after lithium/pilocarpine-induced seizures gradually became obese over their lifetimes if spontaneous seizures developed. Mild increases in weight gain were induced when prepuberal females were given pilocarpine and acepromazine while being exposed briefly $(1.5 \mathrm{hr})$ to a frequency-modulated magnetic field known to induce seizures. Weekly (1.5 hr) exposures to physiologically-patterned magnetic fields over $36 \mathrm{wks}$ had no effect on weight gain while continuous periseizure exposure to $50 \mathrm{~Hz}$ fields above about $1 \mu \mathrm{T}$ facilitated mild weight gains and protracted aggression. Perinatal exposure to a very weak, a $7 \mathrm{~Hz}$ magnetic field or a nitric oxide inhibitor retarded the weight gain induced by the obesity procedure. These results indicate that synergisms during a single episode between neuronal electrical lability and pharmacological states can initiate a process of weight gain that progresses to extreme obesity. We suggest that at least a component of the global "epidemic of obesity" could be related to a synergism between the insidious emergence of amplitude modulations within biologically compatible electromagnetic frequencies from the proliferation of communication systems and the pervasive utilization of pharmacology to treat transient disorders of ontogeny within the human population.
\end{abstract}

\section{Keywords}

Obesity, Drug-Induce Obesity, Weak Magnetic Fields, Drug-Magnetic Field Synergisms 


\section{Introduction}

The etiology of the reported epidemic of obesity within the human population is unclear. If the history of science is generalizable, the global weight gains within the human population are likely to be multivariate. In the following experiments we explored the hypothesis that slow but insidious increases in body weight in female rats could be related synergistically to exposures to environmentally-relevant, weak magnetic fields and pharmacological treatments. Both classes of stimuli are becoming epidemic within the twenty-first century global population [1].

More than thirty years ago, Thomas, Burch, and Yeandle [2] reported a remarkable synergistic effect between microwave radiation and chloradiazepoxide upon fixed-interval behavior in rats. Since that time, numerous classes of pharmaceutical agents such as antidepressants, neuroleptics, benzodiazepines, and compounds designed to affect nitric oxide (NO) pathways have been employed to treat more and more human beings. In addition to the multiple electromagnetic sources within the home environment [3], communication transmissions to support computer systems, the World Wide Web, cell phones, and ancillary electronic devices now produce a ubiquitous field of myriad $\mathrm{MHz}$ to $\mathrm{GHz}$ transmissions whose beat frequencies can occur within the range of neuronal function [4] [5].

While studying the neuromechanism producing pseudocyesis, St-Pierre and Persinger [6] seized prepuberal female rats with a single systemic therapeutic dosage of lithium ( $3 \mathrm{mEq} / \mathrm{kg})$ followed four hours later by a single subcutaneous injection of $30 \mathrm{mg} / \mathrm{kg}$ of pilocarpine [7]. Following the onset of over motor limbic seizures [8], rats were injected with either ketamine $(100 \mathrm{mg} / \mathrm{kg})$ or acepromazine $(25 \mathrm{mg} / \mathrm{kg})$. The same compound produced severe hypothermia when male rats were restrained in previous studies [9]. However we found that only female rats between 30 and 55 days of age that administered this combination of pharmacological compounds began to gain weight. After a year some of these rats weighed more than $1000 \mathrm{~g}$ [6].

Subsequent studies indicated that the female rats who displayed this insidious weight gain over their lifetimes due to this one single "traumatic" episode were the same animals that developed spontaneous seizures a few weeks after the initial seizure induction. This observation was congruent with the effects of extended kindling within the basolateral amygdala [10] and lesioning of the posterodorsal regions of the media amygdala [11] except that the gains were more gradual and continuous over the rat's lifetime.

In the present study, we exposed prepuberal female rats before or after the induction of the obesity procedure to various magnetic field environments. The "obesity procedure" is the term that we have adopted for convenience to describe induction of seizures by lithium/pilocarpine and immediate treatment with acepromazine in prepubescent female rats. We only selected those magnetic field patterns and procedures that we have found produce statistically significant and clinically relevant effects.

These procedures included: 1 ) synergistic effects of perinatal exposure to a NO (nitric oxide) inhibitor in the water supply and a $5 \mathrm{nT}, 7 \mathrm{~Hz}$ magnetic field; this combination resulted in significant juvenile mortality of rats in which lithium/pilocarpine seizures were induced [12]; 2) brief exposures to weak (1 $\mu$ Tesla or $10 \mathrm{mG}$ ) frequency-modulated fields as substitutes for either the lithium or pilocarpine injection; 3) pre- and post-exposure to different intensities between $5 \mathrm{nT}$ and $1.3 \mathrm{uT}$ of an approximately $50 \mathrm{~Hz}$ magnetic field; and 4) weekly exposures, once the obesity procedure had been instituted, to patterns known to induce analgesia or to affect emotional learning.

\section{Methods and Materials}

\subsection{Subjects}

A total of 235 albino Wistar female rats were subjects. They were all offsprings of parents that had been obtained from Charles River, Quebec. The mothers of the subjects had been involved in other experiments.

\subsection{General Procedure}

All protocols were approved by the local animal care committee and followed the guidelines of the Canadian Council on Animal Care. The general procedure was maintained for all experiments unless specified otherwise. The treatment to initiate weight gain began when the rats were between 35 and 51 days of age. Rats were first injected subcutaneously with $3 \mathrm{mEq} / \mathrm{kg}$ of lithium chloride ( $3 \mathrm{mEq} / \mathrm{cc}$ ) into the hind flank.

Four hours later, $30 \mathrm{mg} / \mathrm{kg}$ of pilocarpine (30 mg/cc) was injected (sub. cu.) within the other flank. Seizure 
onset time, defined as rearing and rapid forelimb clonus similar to Racine Type V seizures [8], was recorded to the nearest second. Immediately after the onset of the seizures that only occurred in rats that received the combination of lithium and pilocarpine, they were injected subcutaneously with acepromazine (Atravet, $30 \mathrm{mg} / \mathrm{kg}$ ) in order to facilitate survival. Henceforth, this procedure will be called the obesity (induction) procedure.

Other rats from the same litters were injected with lithium, pilocarpine, or acepromazine only or served as non-injected controls. During the subsequent $72 \mathrm{hr}$, rats were monitored in wire cages because it had been demonstrated in two experiments that placement in plastic cages produced unacceptable mortality even with our treatments. After $72 \mathrm{hr}$, spontaneous mortality was minimal.

Rats were then maintained in groups of 2 or 3 from different treatment conditions within either standard plastic cages or wire cages for the remainder of their lives. They were weighed between once per week to once per month, depending upon the experiment, for up to 14 months. All rooms in which the rats lived were maintained at 20 deg C. The light: dark cycle was 12:12 and began at $08 \mathrm{hr}$. Purina rat chow and water were available Ad libitum.

\subsection{Experiment 1: Spontaneous Seizures and Comparison of Different Post Seizure Compounds}

Previous studies indicated that rats that displayed conspicuous spontaneous seizures during casual observation or after handling during routine cleaning of cages gained more weight. In the first part of this study, the monthly weights were recorded for the rats that received the obesity procedure and that either displayed $(n=4)$ or did not display $(n=5)$ spontaneous seizures during the subsequent year. Non-seized litter mates $(n=5)$ were employed as controls.

Although our previous research indicated that post-seizure injection of acepromazine $(30 \mathrm{mg} / \mathrm{kg})$ but not ketamine $(100 \mathrm{mg} / \mathrm{kg})$ facilitated gradual weight gain and obesity, the second part of the experiment was designed to confirm the requirement for acepromazine and to discern the post seizure effects from a single injection of either doxepin $(10 \mathrm{mg} / \mathrm{kg})$, prazosin $(5 \mathrm{mg} / \mathrm{kg})$ or imipramine $(10 \mathrm{mg} / \mathrm{kg})$. Except for acepromazine, all pharmacological compounds had been obtained from Sigma. A total of 48 rats (8 per group) were injected with one of these drugs after seizure induction. A sixth group served as non-seized controls. Because all but 2 of the imipramine-injected rats died within $72 \mathrm{hr}$ after the seizure induction, the survivors were not included in this study. Six rats from each of the other five conditions were weighed 1, 2, 3 and 6 months after the treatments.

\subsection{Experiment 2: Reactive Hypothermia and Obesity}

Restraint following the same treatment that results in obesity in prepuberal female rats has been shown many times to evoke marked hypothermia (core temperatures as low as 27 deg C) in male seized (and restrained) rats. Depending upon the severity of the hypothermia during the subsequent $24 \mathrm{hr}$ significantly different patterns of brain damage emerge [9]. To discern if the degree of hypothermia could affect the progression of obesity, 8 rats were restrained for $24 \mathrm{hr}$ after administration of the obesity procedure as described by [9]. Rectal core temperature was then taken. An additional 2 rats randomly selected from the same litters served as body weight controls.

\subsection{Experiment 3: Maternal Head Injury}

The contribution of maternal distress to subsequent obesity in the offspring has been considered a low probability but popular hypothesis. Epigenetic effects are known to occur [13]. To test if the physiological consequences of a single mechanical impact to the skull of female rats can be communicated to the later conceived fetus such that the trauma is reflected in the daughters as obesity, 90 to 120 day old female rats were administered the procedures described by Lado and Persinger [14]. Fifteen females were held for 5 sec while a 200 gm weight was dropped from $0.9 \mathrm{~m}$ onto the right side of the skull. The impact velocity is about $15 \mathrm{~km} / \mathrm{hr}$ with a force of about $2 \mathrm{~N}$. The calculated value of the pressure wave under the impact area (diameter $=3 \mathrm{~cm}$ ) was between 3 and 20 $\mathrm{kPa}$ with dorsal-to-ventral transit time of 2 to 3 msec.

This procedure produces stunning, defined as immobility but no loss of reflexes and no "unconsciousness" for about $30 \mathrm{sec}$ in about half the number of rats. The other rats do not show any obvious behavioral alterations. Histological analyses show conspicuous clusters of neurons with shrunken soma within the cortices beneath the impact and within the ventral ("countercoup") limbic structures in both hemispheres. Quantitative estimates suggest that: 1 ) between 10,000 and 50,000 neurons within the cerebral cortices only show this profile (none are apparent in controls) which remains for more than 30 days after the impact; and, 2) the numbers of shrunken 
neurons are not related to the degree of stunning. Immediately after the impact or control condition, half the numbers of rats in each condition were placed with a male or with a female in standard wire cages.

The litters were delivered and maintained with their mothers in standard plastic cages with one-quarter inch Bedocob until they were 21 days of age. The female (offspring) rats were housed, two to three rats per cage, in either wire (standard racks) or plastic cages. Rats randomly selected from the same litters assigned to the obesity procedure $(n=11)$, control group $(n=15)$, or administered either an injection of $3 \mathrm{mEq} / \mathrm{kg}$ of lithium (4), 30 $\mathrm{mg} / \mathrm{kg}$ of acepromazine (4), or $30 \mathrm{mg} / \mathrm{kg}$ of pilocarpine (4) singly. Since there were no differences in weights between the single treatments, they were combined to a single group. Consequently there were three groups of treatments. Body weights were taken 2, 3, 5, and 6 months later. Analyses of variance for body weights a function of whether or not the mother sustained the mechanical impact or not and whether or not the mother was stunned or not stunned were completed. The potential effect of cage condition (wire vs. plastic) upon the gain in body weight was also discerned.

\subsection{Experiment 4: Perinatal Exposure to Environmentally-Patterned Magnetic Fields and Nitric Oxide Inhibitors}

That perinatal and postnatal conditions can produce long-term effects upon behaviour and physiology is well established. A total of 12 pregnant female rats (about 90 days old) had been exposed from 2 days before birth to 14 days after birth to a $7 \mathrm{~Hz}, 5 \mathrm{nT}$ sine wave magnetic field or sham condition and given either normal tap water or tap water with $0.5 \mathrm{~g} / \mathrm{L}$ of methyl arginine in the water supply. Although this field strength is very weak, it has been shown to affect mortality of post-weaned rats when they are seized [12]. At weaning the rats were housed in plastic cages.

Because synergistic interactions and cancellations between this dosage of methyl arginine and this field strength upon organ weights when these rats become adults have been shown the design was a three way analyses: obesity procedure (no, yes), exposed to the magnetic field (no, yes), or exposed to the NO inhibitor (no, yes) in the water supply. There were 8 to 9 rats per group $(n=51)$. Half the numbers of rats from each of the six groups were maintained in either plastic or wire cages. Weights were taken at 4 and 6 months after the seizure induction.

\subsection{Experiment 5: Single Pairing of Frequency-Modulated Magnetic Field with Pharmacology}

The emergence of progressive obesity following the synergistic action of prepuberal limbic seizures and specific post-seizure neuroleptic compounds is a powerful effect but its relevance to human settings would be limited to individuals with temporal lobe electrical lability or subclinical complex partial seizures. In addition there is multifocal damage within the brains of rats seized by using the pilocarpine or lithium-pilocarpine method. Previous studies have shown that exposure for one hour to a specific [5] frequency modulated magnetic field that produces analgesia in rats [15] and snails [16] [17] increases the probability of limbic motor seizures in seized rats. Longer exposures during labile periods such as early development produce permanent changes in specific organ weights [18].

To discern if exposure to this seizure-inducing magnetic field might produce similar progressive weight gains if applied at the time of excessive muscarinic cholinergic stimulation, rats that were administered the obesity procedures were compared to rats that were given the dosage of pilocarpine or lithium only and then exposed to the frequency-modulated field known to evoke seizures in rats with histories of lithium/pilocarpine-induced epilepsy. Following cholinergic induction of seizures, more than 50\% of CA pyramidal cells which normally fire in a regular mode are persistently converted to a bursting mode because of up-regulation of T-type calcium channels [19]. Here we examined if the muscarinic transient muscarinic state would synergistically interact with the applied burst-firing and frequency-modulated field to produce an electrical lability that would simulate electrical seizures and hence contribute to obesity.

The experiment was completed in two blocks. The first block of rats was maintained in plastic cages while the second block was maintained in wire cages. In each block the groups were given the obesity procedure $(n=9)$, or, either the pilocarpine only $(n=4)$ or lithium $(n=4)$ only, injected with acepromazine one hour later, and then exposed to the frequency-modulated magnetic field. A fourth group served as controls $(n=4)$. Hence the total sample was 42 . 
The two groups in each block exposed to the magnetic field were placed in the "4-D chamber" described previously [20]. It is a plastic box with pairs of solenoids in each of the three spatial planes. The magnetic field pattern was presented for $0.5 \mathrm{sec}$ in each of the three planes and then in all three planes such that the total cycle time was 2 sec. A picture of the frequency-modulated magnetic field or "Thomas pulse" has been published previously [21]. It was generated by custom-constructed software through a digital-to-analogue converter that delivered the fields successively to the pairs of solenoids. The duration of the each point between $-5 \mathrm{~V}$ and $+5 \mathrm{~V}$ was $3 \mathrm{msec}$, a value that is important for effects in both cells [22] and human subjects [23].

The total exposure duration was $1.5 \mathrm{hr}$ and started immediately after the injection of acepromazine. The range of magnetic field intensity within the exposure area was between 0.8 and $1.2 \mu$ Tesla with an induced electric field, according to Faraday's law and using our parameters within a $\mathrm{cm}^{2}$ region, in the order of $10^{-6} \mathrm{~V}$. According to Adey's [24] modifications of the Boltzmann equation that model neuronal tissue as a low-pass filter, $\mathrm{V}^{2}$ (voltage) is the product of $4 \mathrm{kTBR}$ where $\mathrm{k}$ is the Boltzmann constant, $\mathrm{T}$ is temperature, $\mathrm{B}$ is the frequency band, and $\mathrm{R}$ is the specific resistance of the noise pathway which for brain tissue is in the order of $300 \mathrm{ohm} \mathrm{cm}$. Although this potential difference is small it is still above the equivalent (temperature-related) noise voltage gradient of $10^{-8} \mathrm{~V} / \mathrm{cm}$. Sensitivities in some marine vertebrates are within this range. It may be relevant that the voltages associated with reconnection in geomagnetic flux lines following alterations in the solar wind (and geomagnetic activity) are in the order of $4 \times 10^{-6} \mathrm{~V} / \mathrm{cm}[25]$.

The estimated resultant voltage from the applied fields, of about $10^{-7} \mathrm{~V} / \mathrm{cm}$, would be sufficient to be detected after $10 \mathrm{msec}$ by an ensemble of less than $10^{8}$ ion channels [26] distributed over a structure, such as the hippocampus, dominated by electrical interfaces [27] and characteristics that facilitate phase coupling by synaptic spread of coupled neuronal oscillators [28]. These intensities and this duration of exposure are sufficient to produce very robust changes in nociceptive thresholds and learning [15]. Weights were taken frequently in order to see the time of emergence of any significant gains.

\subsection{Experiment 6: Protracted Exposures to a Power-Frequency Magnetic Field}

In the present experiment rats were exposed from 5 days before to three weeks after the obesity procedure to a custom constructed magnetic field, generated by computer software, to "interfere" with 20 base long RNAi (inhibiting RNA). We assumed that the $10^{-20} \mathrm{~J}$ of energy [29] [30] associated with the stacking of one base requiring about 1 msec could be modified if 10, 1 msec pulses each separated by 1 msec were generated continuously. However the net effect (total pulse train duration of about $20 \mathrm{msec}$ ) was effectively a $50 \mathrm{~Hz}$ square-wave field. It differed from a classic function generated field because each point duration was $1 \mathrm{msec}$ that was "recreated" every 1 msec by the computer processor.

Such fields when applied within the $10 \mathrm{mT}$ range can affect cytosolic $\mathrm{Ca}^{++}$levels [31]. As indicated by a variety of studies that were first demonstrated by Adey [24], weaker, fields within this frequency band can facilitate neuronal recovery. Ishido, Nitta and Kabuto [32] found that $1.2 \mu \mathrm{T}, 50 \mathrm{~Hz}$ magnetic fields uncouple inhibitory pathways of adenyl cyclase mediated by the melatonin 1a receptor in specific cell lines. Melatonin prevents the delayed death of hippocampal neurons induced by enhanced excitatory neurotransmission and NO pathways [33]. Melatonin levels, which are well known to be affected by weak magnetic fields, in gastrointestinal tissue surpass blood concentrations by a factor of 100 [34] [35].

In two separate blocks of experiments pairs of rats were exposed continuously (except for 5 min during cage cleaning) to one of four intensities of magnetic fields as described by St-Pierre et al. [21] [36]. These intensity ranges per group were very low (5 - $20 \mathrm{nT})$, low (30 - $50 \mathrm{nT})$, medium (90 - $580 \mathrm{nT})$ and high (590 nT to 1.2 $\mu \mathrm{T}$ ). Total numbers of rats were 16 (4/intensity). When the 26 days of exposure had elapsed the rats were maintained in pairs (from the same exposure condition) in standard plastic cages. Weights were taken twice per month. The emergence of aggressive behaviour was monitored several times per day (starting at $07 \mathrm{hr}$ ) and night until $03 \mathrm{hr}$. Aggression as defined by any cut on the cage mate or observation of agonistic behaviour, not typical of this strain of albino female rat, was recorded.

\subsection{Experiment 7: Weekly Exposures to One of Two Physiologically-Patterned Fields upon the Rate of Weight Gain}

This experiment was designed to discern if once rats had begun the progressive growth towards obesity the rate could be diminished or inhibited by weekly $(1.5 \mathrm{hr})$ treatments with physiologically-patterned magnetic fields. 
Two fields were selected. The first group $(n=5)$ received a burst-firing field that is known to simulate the effects of opiates [15]; its pattern has been published elsewhere [17]. The second group $(n=6)$ received a pattern, that when applied as a direct current into hippocampal slices, has been shown to affect the development of long-term potentiation (LTP). A third group $(n=4)$ of rats served as non-treated, sham field-exposed controls.

The burst-firing field contained point durations of $3 \mathrm{msec}$ with a total duration of $1 \mathrm{sec}$ separated by $4 \mathrm{sec}$ of no stimulation. The point durations $(1 \mathrm{msec})$ of the second pattern involved repeated presentations of a priming pulse for 2 msec followed 170 msec by four $100 \mathrm{~Hz}$ pulses and then $4 \mathrm{sec}$ of no stimulation [37]. The patterns were presented sequentially in each of the three spatial planes and then in all planes (one cycle $=2 \mathrm{sec}$ ) within the $4 \mathrm{D}$ chamber. The magnetic field or sham treatments began two months after the initiation of the obesity procedure and continued for 36 weeks. Weights were taken for an additional three months after the termination of the treatment.

\section{Statistical Analyses}

The primary designs were multifactorial, mixed analyses of variance which included both between (treatments) and within (repeated measures) subject levels. Post hoc analyses employed one-way analyses of variance and post hoc (primarily Tukey's and Scheffe's) to discern the source of significance for multiple group designs. Where within- and between-subjects interactions occurred, a combination of selective paired t-tests and oneway analyses of variance were combined. When issues of homogeneity of variance occurred, nonparmetric procedures, particularly Kruskal-Wallis were employed for verification. Scattergrams and correlations were completed to visualize the data. All analyses involved SPSS 16 for PC. Eta-squared estimates (or omega-squared estimates) for effect sizes, which reflect the amounts of variance in the dependent measure accommodated or "explained" by the experimental manipulation or level were included to demonstrate the potential applicability of the effects.

\section{Results}

\subsection{Experiment 1}

The means and SEMs for the pretreated body weights and the progressive increase in body weights per month over the subsequent year for rats that received the obesity procedure and either displayed spontaneous seizures or did not are shown in Figure 1. Weights of non-seized controls are shown for comparison. Analyses of variance (all dfs $=2,11$ ) demonstrated the significant increase in body weight in the rats that ultimately showed spontaneous seizures within one month. There was no overlap in body weights between this group and the other two groups by the second month. The significant correlations $(r>0.70)$ between the numbers of observed spontaneous seizures (range 0 to 6 ) and body weight emerged by the eight month and remained stable.

The means and SEMs for the rats that served as non-seized controls or that were seized and immediately received either acepromazine (the obesity treatment), ketamine, prazosin, or doxepin, are shown in Table 1 . One way analyses of variance (all dfs $=4,25)$ showed statistically significant $(\mathrm{p}<0.01)$ group differences one $\left(F=5.26\right.$; eta $\left.^{2}=46 \%\right)$, two $\left(F=14.86\right.$, eta $\left.^{2}=70 \%\right)$, three $\left(F=12.83\right.$, eta $\left.{ }^{2}=67 \%\right)$ and six $\left(F=31.40\right.$, eta $\left.{ }^{2}=82 \%\right)$ months after the treatments. Post hoc analysis indicated that the main source of the effect was due to heavier weights of the rats that received the acepromazine (the obesity procedure) compared to other post-seizure treatments that did not differ significantly from each other. It may be relevant that no spontaneous seizures were noted in any of the groups except those treated with acepromazine.

\subsection{Experiment 2}

The means and SEMs for the rats $(n=6)$ that displayed at least one spontaneous seizure during cage-cleaning or weighing 2, 4, and 6 months after receiving the obesity treatment and then physically restrained were 305 (9), 449 (9), and 494 (13), respectively and were significantly heavier $[F(1,8)=5.74,15.01,11.42$, p < 0.01; explaining between $42 \%$ and $64 \%$ of the variance] while the comparable weights for those that did not $(n=4)$ spontaneously seizures were 270 (12), 358 (25), and 389 (32). The reference controls' $(n=4)$ weight during this period ranged from 272 (10) to 310 (13).

The pre-treatment weights for the spontaneous seizing and non-seizing rats were 169 (9) and 157 (5), respec- 


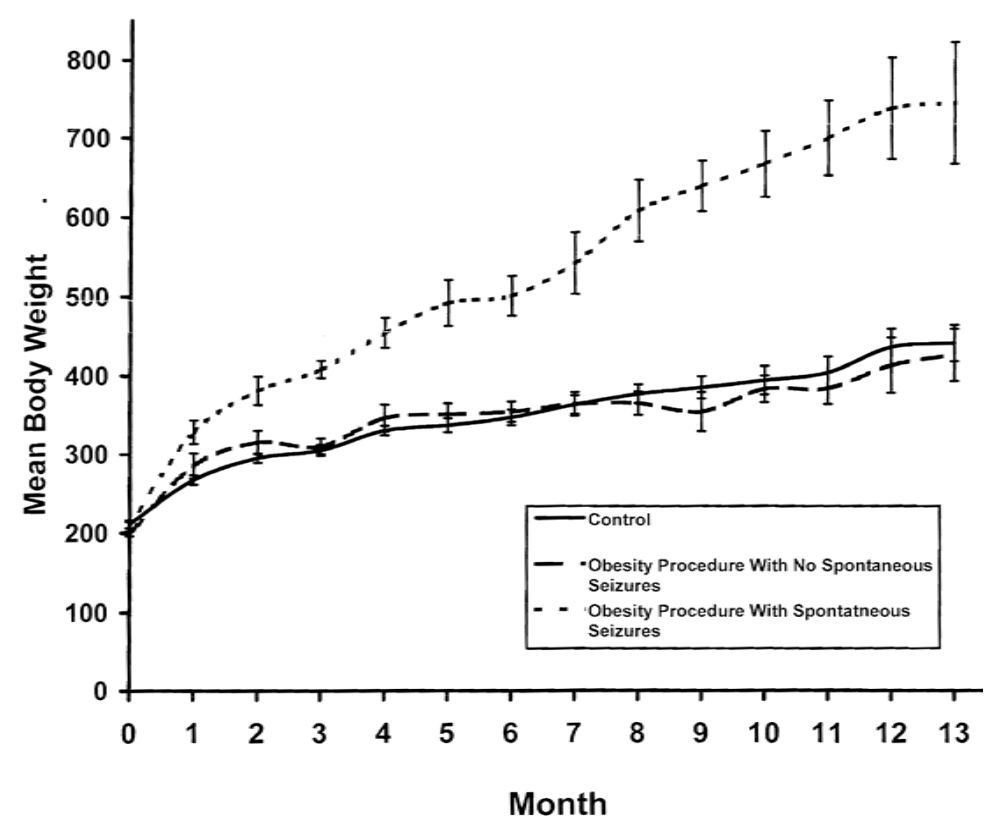

Figure 1. Mean body weight (gm) for groups that received the obesity procedure and did or did not display overt spontaneous seizures sometime during the subsequent 13 months. The control group were non-seized litter mate controls. Vertical bars indicate SEMS.

Table 1. Means and standard errors of the mean (SEM) for body weights over several months (in gm) of female rats that were litter mate controls or that were seized with lithium/pilocarpine and treated immediately with either acepromazine, prazosin, ketamine, or doxepin.

\begin{tabular}{ccccccccccccc}
\hline Month & \multicolumn{2}{c}{ Control } & \multicolumn{2}{c}{ Doxepin } & \multicolumn{2}{c}{ Prazosin } & \multicolumn{2}{c}{ Ketamine } & \multicolumn{3}{c}{ Acepromazine } \\
\hline & M & SEM & M & SEM & M & SEM & M & SEM & M & SEM \\
\hline 0 & 215 & 5 & 217 & 10 & 222 & 8 & 210 & 6 & 221 & 5 \\
1 & 254 & 9 & 249 & 13 & 271 & 16 & 274 & 3 & $322^{\mathrm{b}}$ & 16 \\
2 & 298 & 12 & 289 & 10 & 298 & 16 & 302 & 6 & $396^{\mathrm{b}}$ & 12 \\
3 & 316 & 18 & 308 & 16 & 310 & 12 & 319 & 6 & $435^{\mathrm{b}}$ & 19 \\
6 & 348 & 23 & 365 & 20 & 348 & 13 & 333 & 5 & $623^{\mathrm{b}}$ & 35 \\
\hline
\end{tabular}

${ }^{\mathrm{b}}$ indicates this group was significantly different from all other groups.

tively, did not differ significantly $[F(1,8)=1.49, \mathrm{p}>0.05]$. The body hypothermia ranged between 33.7 and 37 degrees $\mathrm{C}$. The Spearman correlations between weight and degree of transient hypothermia after seizure induction for the 2, 4, and 6 months later were $-0.08,-0.52(\mathrm{p}<0.05)$ and $-0.73(\mathrm{p}<0.01)$ indicating that the more severe hypothermia was associated with more significant weight gain.

\subsection{Experiment 3}

The means and SEMs (in parentheses) for the body weights 2, 3, and 5 for rats that were controls $(n=11)$, received the obesity procedure (15), or single drug treatments $(n=13$, only injected with either acepromazine, lithium, pilocarpine, or ketamine) were 280 (9), 442 (27), 307 (6) gm, for month 2, 296 (11), 489 (30), 325 (6) gm for month 3, and 5: 307 (12), 519 (37), and 338 (8) gm for month 5, respectively. There were significant group differences over these periods $[\mathrm{Fs}(2,38)=20.92,24.81,21.14$, $\mathrm{p}<0.001 ; 50 \%$ to $58 \%$ of variance explained].

Post hoc analyses indicated rats that received the obesity procedure remained significantly heavier than the 
non-seized or single-treatment only litter mates. Bartlett Box results (all Fs > 15.00) indicated significantly more variability in the seized + acepromazine (obesity procedure) group than in the other two groups. However Kruskal-Wallis, non-parametric analyses indicated the group differences were still strong and statistically significant.

Analysis of variance indicated no significant contribution from whether or not pregnancy and motherhood followed the head trauma $[\mathrm{Fs}(1,38)<1]$. For the off springs $(n=13,6)$ of mothers that had displayed stunning or not stunning, respectively, there were no significant difference in body weights for any of the measurement period. Similarly being housed in either plastic cages or wire cages over several months did not differentially significantly affect the weight gains in any of the groups as indicated by the absence of a significant interaction $[\mathrm{F}<1.00]$ between treatment and cage condition. Chi-squared analyses for the numbers of spontaneous deaths or the 5 months of observation was $27 \%$ for those housed in plastic cages and $9 \%$ for those house in wire cages (chi-squared $=3.14, \mathrm{p}=0.07$ ).

\subsection{Experiment 4}

Three-way analyses of variance as a function of perinatal magnetic field exposure ( $5 \mathrm{nT}, 7 \mathrm{~Hz}$; sham), NO inhibitor treatment in the maternal water supply (no, yes) and administration of the obesity treatment (no, yes) at 53 days of age for body weights 2 months and 4 months later demonstrated statistically significant main effects (all dfs $=1,53$ ). The rats that had been exposed to the magnetic field $[\mathrm{F}=6.48,4.47, \mathrm{p}<0.05$; eta $=0.27,0.21]$ or to the NO inhibitor $[\mathrm{F}=11.21,13.41 \mathrm{p}<0.001$; eta $=0.36,0.36]$ weighed less than their controls. However the rats that had received the obesity procedure as adults were significantly heavier $[\mathrm{F}=27.81,43.67, \mathrm{p}<0.001$; eta $=0.56,0.64]$ than those that had not.

However by 4 months post-obesity procedure there was a statistically significant interaction between field and seizure conditions $[\mathrm{F}=7.85, \mathrm{p}<0.01]$ and between drug and seizure conditions $[\mathrm{F}=5.81, \mathrm{p}<0.05]$. Post hoc analysis for the first interaction is shown in Figure 2 (where month 2 and 4 are indicated as month 1 and 2, respectively). The perinatal magnetic field treatment diminished the magnitude of the weight gain of the rats that had been given the obesity procedure. Post hoc analysis of the interaction between receiving the NO inhibitor in the mother's water supply and obesity procedure showed a similar effect. The rats whose mothers received the NO-inhibitor showed less weight gain. The effects of the perinatal magnetic field $[F(1,41)=5.45, p<0.05]$ upon slowing weight gain were still evident at six months post obesity procedure even though the population of rats had diminished. The mean discrepancy between the seized rats and non-seized litter mate controls was 102 gm at that time.

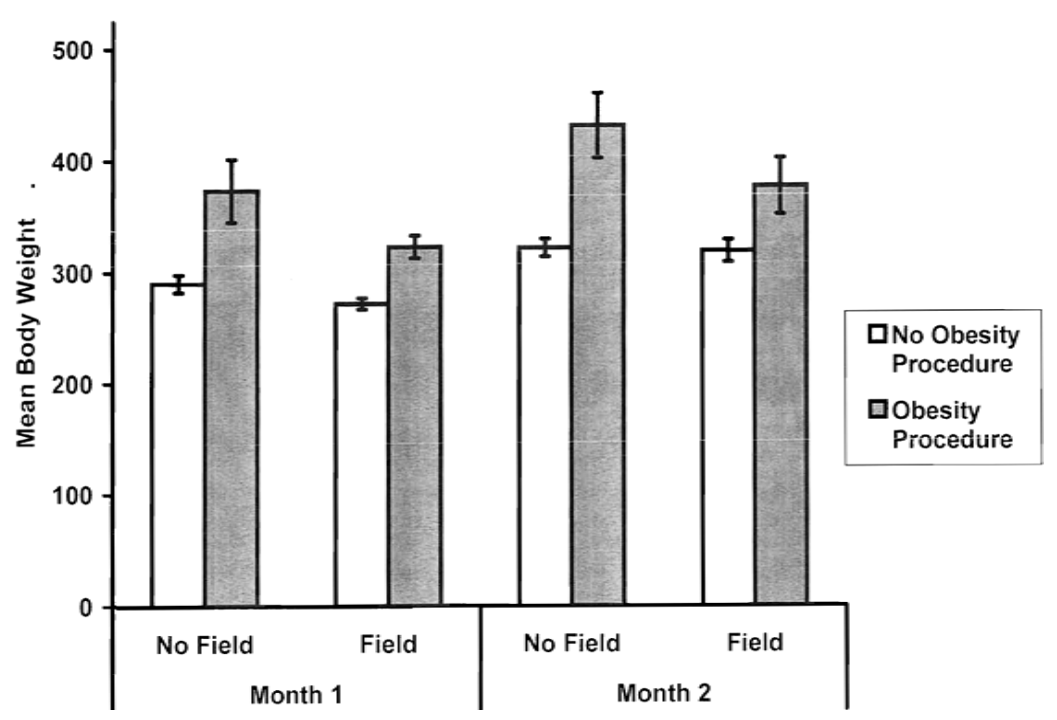

Figure 2. Mean body weights (gm) for groups one and two months after they did or did not receive the obesity procedure as a function of perinatal exposure (no, yes) to a weak (5 nT), $7 \mathrm{~Hz}$ magnetic field known to interact with NO treatments. Vertical bars indicate SEMs. 
There were spontaneous deaths within this population. Hierarchical log-linear analyses, verified by three-way analysis of variance, indicated that the rats $(n=5)$ that had received the combination of the obesity procedure, the magnetic field, and the NO-inhibitor died suddenly during the 6 month observation period. They were synergistic-induced spontaneous deaths.

\subsection{Experiment 5}

For block 1, the means and SEMs (in parentheses) of the weights two months later for the groups that received the various treatments were obesity 322 (9) gm, control 264 (2) gm, lithium only + magnetic field + acepromazine 270 (2) gm and pilocarpine only + magnetic field + acepromazine 288 (14) gm. The source of the statistically significant $[\mathrm{F}(3,17)=12.24, \mathrm{p}<0.001$; eta-squared $=72 \%]$ group difference was due to the heavier body weights for the rats that received the obesity procedure compared to other three groups and the group that received the pilocarpine + magnetic field + acepromazine compared to the other two groups.

For block 2, the means and SEMs for body weights for the day of the treatments and 1, 2, 3, 4, and 5 months afterwards for the groups that were controls or received the obesity procedure, lithium only + magnetic field + acepromazine, or pilocarpine only + magnetic field + acepromazine, are shown in Figure 3. Analyses of variance (all dfs $=3,12$ ) demonstrated significant differences only on months $2[\mathrm{~F}=4.36]$ and 3 [ $\mathrm{F}=3.94]$. However, Kruskal-Wallis analyses indicated group differences were present for months 2 (chi-squared $=9.72$ ), 3 (chi-squared $=11.32), 4$ (chi-squared $=8.77)$.

Post hoc analysis indicated that the major source of the group difference was between the group that received the obesity procedure and the other three groups. A secondary effect was evident as heavier weights for the pilocarpine + magnetic field + acepromazine group compared to the lithium + magnetic field + acepromazine group.

\subsection{Experiment 6}

The mean body weights between the groups of rats exposed to the four different magnetic field different intensities at the time of the obesity procedure did not differ significantly $[F(3,12)=0.11, p>0.05]$ from each other (grand mean and S.E.M = 184.8, 6.5 gm, respectively). Within one month the usual accelerated weight gain was evident. However there was a significant linear trend $[F(1,12)=5.62, p<0.05]$ for less weight gain with increased magnetic field intensity (Figure 4). This weak but statistically significant effect of inhibiting the weight gain as a function of increased field intensity continued for about two months. After that time the within group measures of dispersion increased and the effect diminished.

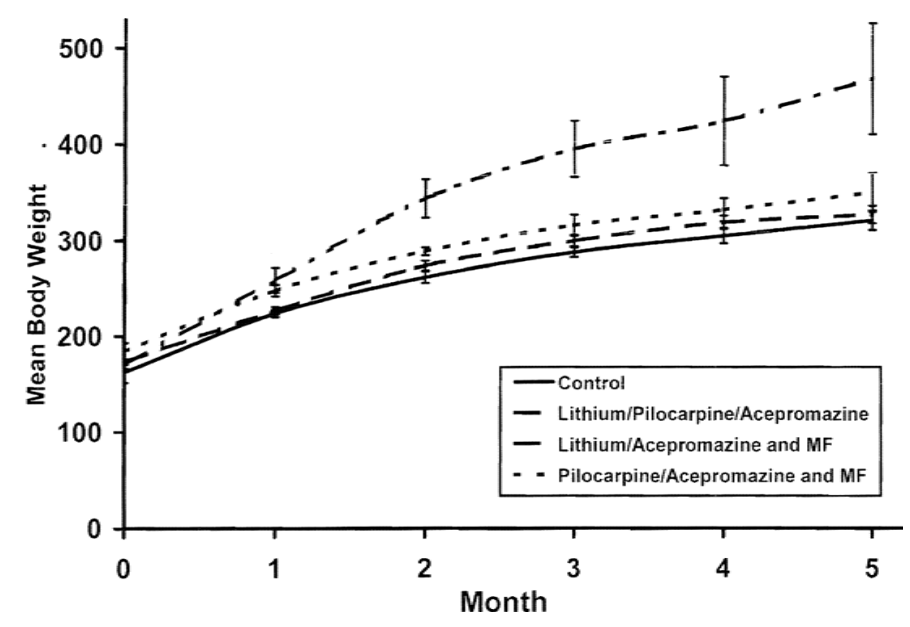

Figure 3. Mean body weights (gm) over 5 months for groups that received the obesity procedure or were exposed to either lithium + acepromazine or pilocarpine + acepromazine in the presence of a frequency-modulated magnetic field known to induce seizures in epileptic rats. Controls were non-treated litter mates. Vertical bars indicate SEMs. 


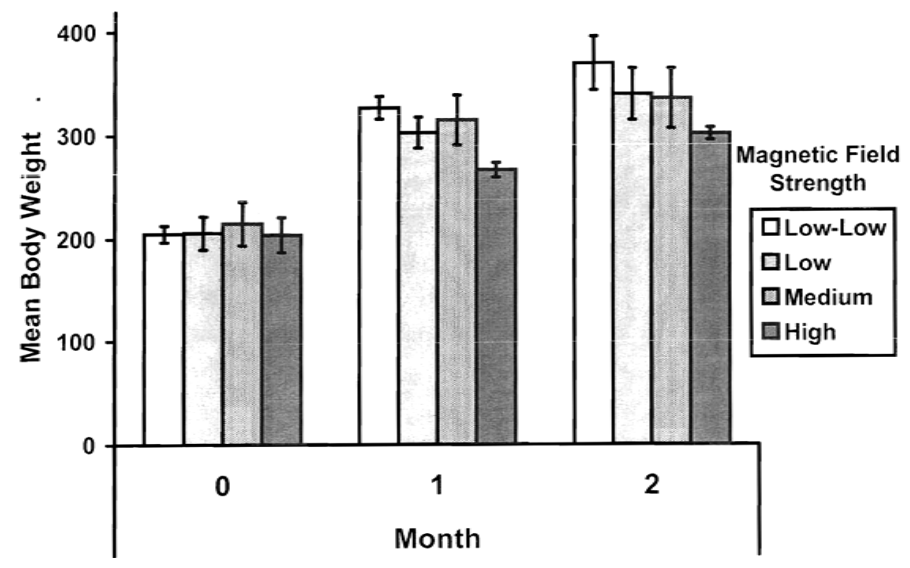

Figure 4. Mean body weights (gm) during the first and second month for groups exposed to before, during and after the obesity procedure to $50 \mathrm{~Hz}$ magnetic fields of different intensities. Vertical bars are SEMs.

An unexpected phenomenon was the increase of marked agonistic behaviour between pairs of housed rats. This behaviour was qualitatively conspicuous. Although there was no significant difference between body weights of the 8 rats that became the apparent source of the aggression $(M=181, S E M=8)$ and the 8 rats that appeared more subordinate $(M=188, S E M=10)$, by the time the aggressive became obvious five months later the aggressive rats were trending toward heavier $(M=477$, SEM $=34)$ body weights than the non-aggressive $(M=397, S E M=20)$. Ten months later the increased body weight in the aggressive females $(M=625, S E M=$ 29) was significantly greater $[F(1,14)=10.20, \mathrm{p}<0.01 ; 46 \%$ percentage of variance explained] than the more submissive cage mates $(M=488, \mathrm{SEM}=31)$. This significant discrepancy was still evident 1.2 years after the obesity procedure.

\subsection{Experiment 7}

The monthly mean weights of the rats that were administered the obesity procedure and then exposed once per week for $1.5 \mathrm{hr}$ to either a burst-firing magnetic field $(\mathrm{n}=5)$, a LTP-inducing pattern $(\mathrm{n}=6)$, or the sham field condition $(n=5)$ are shown in Figure 5. One way analyses of variance (all dfs $=2,13$ ) indicated no significant main effect for group differences $(\mathrm{F}=2.50)$ although several points were individually significant (no overlap of SEMs). The group separations were most evident after the sixth month. The magnetic field treatments were stopped after 9 months (about 36 exposures). The effect was diminished about two months after the treatments were terminated.

\section{Discussion}

The results of these experiments verified previous observations [6] that a specific pharmacological treatment for the electrical lability induced by lithium/pilocarpine seizures contributed to life-long obesity in female rats. Again, treatment by ketamine $(100 \mathrm{mg} / \mathrm{kg})$ rather than acepromazine immediately after the display of the over seizures did not result in the weight gain during subsequent months. Neither post-seizure treatment with doxepin, that had been selected because of its marked cholinergic effects, nor prazosin, which had been selected because of its specific antagonism for the alpha-1 adrenergic receptor [9], resulted in the accelerated weight gain relative to normal controls.

The absence of weight gain in rats treated with prazosin at the dosage that produced severe hypothermia (poikilothermia) associated with acepromazine in male rats [9] suggests that the mechanisms for the hypothermia in males and weight gain in females may be different. It may be relevant that alpha-1 adrenoreceptors are directly involved with norepinephrine induced thermogenesis in non brown adipose tissues. Because these receptors are located on adipocytes and they are affected by hormonal variations, the singular heterostasis of prepubescent seizures may have altered the set point for the brain-adipose axis [38].

That adipose tissue is the source of hormones, such as that leptin, with central actions is well known [39]. Obesity-inducing lesions within the central nervous system, such as the paraventricular nucleus and the posterodorsa 


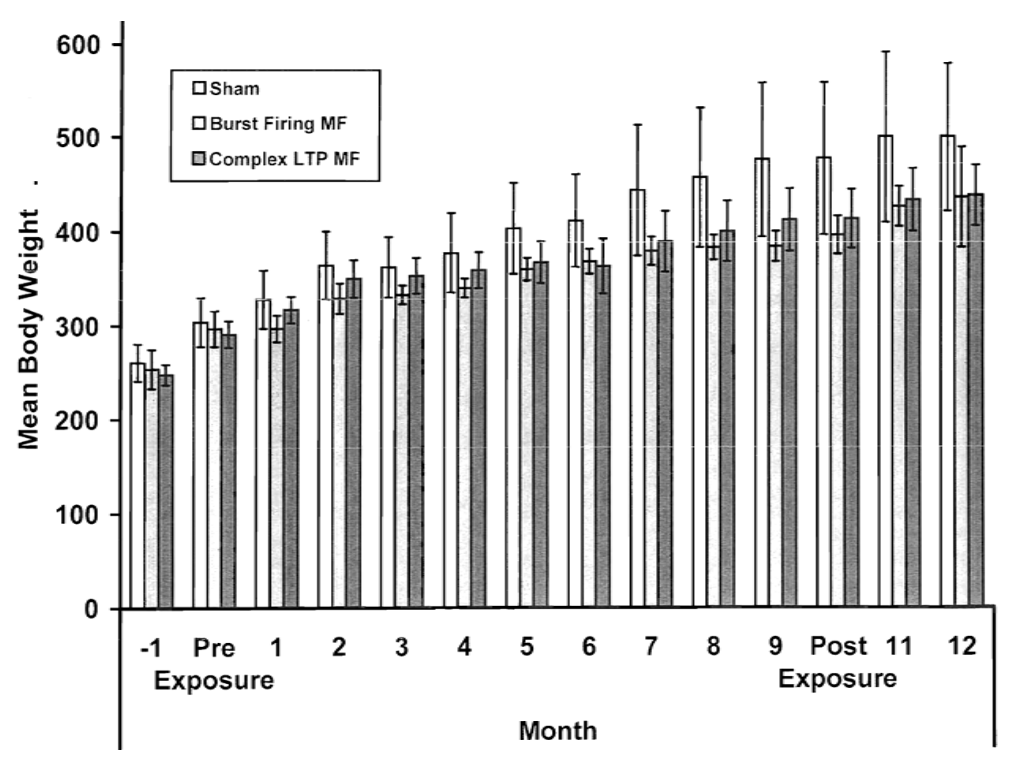

Figure 5. Mean body weights (gm) for groups that were exposed $1.5 \mathrm{hr}$ per week for 9 months after the induction of the obesity procedure to either a sham condition, a burst-firing magnetic known to simulate opiates, or, a magnetic field simulating LTP (long-term potentiation) effects. Body weights for the two months before the treatment and three months after the treatment are also shown. Vertical bars indicated SEMs.

amygdala, alter leptin uptake by the blood-brain barrier [40]. These structures, among others, are clearly damaged in the brains of rats in which seizures have been induced by lithium and pilocarpine [7] as well as in rats seized with only $380 \mathrm{mg} / \mathrm{kg}$ of pilocarpine [41]. In the present study the rats that displayed the greatest hypothermia exhibited the largest weight gains. Male rats that displayed hypothermia, compared to those who did not, exhibited twice the proportion of damage in the basolateral amygdala.

Excessive weight gains in female rats have been induced by extended kindling of the basolateral amygdala [10]. Our post-hoc analyses also verified that only those rats who ultimately displayed the spontaneous seizures, that emerged about 2 to 10 weeks after the seizure induction and at about the time that aberrant mossy fibers from granule cells sprouted into their own dendritic fields, exhibited the massive weight gains. These results indicate that specific electrical lability rather than brain damage per se is the central causative factor for the insidious obesity.

Our attempt to facilitate electrical lability following injection of either lithium or pilocarpine by contiguous whole body application for $1.5 \mathrm{hr}$ to a frequency modulated magnetic field that induces seizures in epileptic rats showed only a transient effect. A small but significant weight gain occurred during the month following this treatment only for the rats that received this field and the cholinergic (pilocarpine) but not the lithium stimulation. This indicates that the electrical lability was not permanent, unlike that associated with the persistent lability reflected occasionally by the overt seizures in the obesity procedure group. The intensities were much larger than the picoTesla/Hz values within the environment [42] but well within the range generated by myriad of electrical devices including computer terminals, telephones, and earphones (or earbuds) from communication devices.

Weak magnetic fields have been shown to induce seizures in human epileptics [43] and it may be relevant that psychogenic non-epileptic seizures are associated with increased risk of obesity [44]. Why only some rats displayed the spontaneous seizures and the obesity is likely coupled to the hemishpericity of damage. Bhatt et al. [45] showed weight gain was four times greater in rats kindled in the left basal amygdala compared to the right. According to Persinger and Dupont [46] the numbers of spontaneous seizures in male rats over a one-year period were positively correlated with the amount of damage in the right temporal cortices but negatively correlated with damage within the left amygdala, a condition that might promote left amygdaloidal lability.

A single $(1.5 \mathrm{hr})$ exposure per week for 36 weeks to either a burst-firing magnetic field known to simulate opioid effects [15] [17] or a field configuration known to induce long-term potentiation when applied to hippo- 
campal slices or powerful inhibition of contextual fear behavior when applied to the whole body [20] exhibited a mild effect. There was a weak diminishing of the rate of weight gain during the latter periods of the exposures. The numbers of repeated measures diluted the statistical significance.

This "mild" inhibitory effect was lost within a month of cessation of exposure. We anticipated that protracted weekly stimulation, a type of long-term homology of kindling, might facilitate the weight gain. That diet-induced obesity increased mu opioid receptor binding occurs in the same specific regions of the brain, the basolateral nucleus [47] that was involved with spontaneous seizures, has been observed. This did not occur. In fact there was a mild suppression of weight gain after about 6 months of weekly exposures.

On the other hand continuous exposure to a $50 \mathrm{~Hz}$ magnetic field produced a clearer although transient decrease in weight gain only for those females exposed to the approximately $1 \mu \mathrm{T}$ field but not to weaker intensities. The effect was not maintained when they were removed from the field. Similar intensities fields have been shown to uncouple the inhibitory pathways of adenylyl cyclase [32]. Hogan and Wieraszko [48] found that hippocampal slices exposed to even $0.16 \mathrm{~Hz}, 15 \mathrm{mT}$ fields for 30 min increased cAMP levels.

In light of the capacity for $60 \mathrm{~Hz}$ magnetic fields [49], to activate MAPK/ERK (mitogen-activated protein kinase or extracelluar signal-regulated kinase), these signalling pathways might be investigated. Recently Buckner et al. [20] found that exposure to the same frequency-modulated magnetic field between $5 \mu \mathrm{T}$ and $10 \mu \mathrm{T}$ inhibited B16-BL6 (mouse melanoma cell) proliferation. The effect was abolished when cAMP-dependent signalling was blocked with SQ22536 or forskolin. cAMP is a primary mediating pathway within cells, including adipocytes, for noradrenergic receptor activation.

The emergence of aggression that was maintained in rats that were exposed to the " $50 \mathrm{~Hz}$ " pattern (that was intended to be the temporal equivalent of RNAi) could be similar to the effects of the frequency-modulated ("Thomas pulse") pattern that have been observed for male rats [36]. The intensity of the agonistic behavior displayed by the females in the present study that had received the obesity procedure has not been observed previously except perhaps during maternal care. Females that were seized with lithium/pilocarpine and given acepromazine as adults (rather than adolescents) do not gain weight over time and show normal fecundity. However they do not care for their young, are extremely aggressive during this period, and engage in infantophagia [50].

In the present study a history of brain trauma, designed to simulate mild head injuries in the human population [14], did not significantly affect the weight gain in the off springs when they received the obesity procedure. Such traumas often result in marked hormonal variations following impact. On the other hand exposure to $5 \mathrm{nT}$, $7 \mathrm{~Hz}$ magnetic fields during early development reduced the rate of weight gain. It may be relevant that other studies have shown alterations in the neuronal/glial densities of the solitary and parasolitary nucleus following perinatal exposure to similar frequency $(0.5 \mathrm{~Hz})$ weak magnetic fields [51]. Both the nucleus of the solitary tract, a closely related structure, and the central nucleus of the amygdala (which is disinhibited by lithium/pilocarpineinduced seizure damage) are important regulators of ingestive behaviors [52].

The possible modulation of potential weight gain following an obesity procedure by different subtle perinatal environments may be relevant. The configurations of weak magnetic fields employed in this study are very likely to be encountered intermittently by human beings. Pulsed magnetic fields significantly affect NO pathways [1]. Consistent with this effect is the current observation that rats exposed to a NO inhibitor during development also gained less weight when challenged with the obesity procedure. Considering the contribution of gastrointestinal peptides, such as leptin and choleocystokin, to the neural-mediated behavior that leads to obesity, the factors that affect this system [34] [35] are critical.

The presence of comparable or "functionally-equivalent" electromagnetic patterns employed in our studies within the modern habitat will require specific measurements. We have found remarkably similar magnetic field patterns generated by a defective digital clock that elicited emotionally significant experiences and altered perceptions within the brain of a young teenage female who had a history of right prefrontal injury [53]. Direct measurements of upper $\mathrm{MHz}$ to $\mathrm{GHz}$ power densities with instrumentation now available from commercial sources routinely indicate that values in the order of 1 to $20 \mathrm{~mW}$ per $\mathrm{m}^{2}$ are common within 5 to $10 \mathrm{~m}$ of many popular brands of microwave ovens when they are operating. Background levels within the sleeping areas of many homes from wireless systems show amplitude modulations, within brain frequency ranges. The power density (radiant emittance) is in the order of 0.5 to $1 \mathrm{~mW}$ per $\mathrm{m}^{2}$.

Assuming complete penetrability of the skull, the energy within one typical neuron with a diameter of $10 \mathrm{mi}-$ crometers would be within the range of a picoJoule per s. This is the same quantity of energy associated with local (intracellular) glucose utilization. Whether or not such juxtaposition of electromagnetic sources and meta- 
bolic derivations of quantum energies would generate an emergent condition that would constitute the "limbic" sensitivity of the type required to produce the insidious obesity measured reliably in our experiments requires further study. However we suggest that the escalation of electromagnetic densities from Western civilization that are now transglobal in nature is conspicuously coincident with the "epidemic" of obesity and the remarkably increased dependence upon pharmacological consumptions for a wide variety of normal challenges associated with ontogeny. Although we do not have direct measurements for the entire population, our clinical experience with a local sample indicates that the proportion of pre- and early post-adolescent females who have been prescribed psychotropic medications at least once exceeds at least about $25 \%$. This value approaches the shift in the distributional curve towards larger body masses.

In summary, the insidious weight gain associated with the administration of acepromazine after lithium/pilocarpine-induced seizures in prepubescent female rats appears to require the occurrence of spontaneous seizures. Applications of magnetic fields that promoted "spontaneous" seizures (in previous studies) in seized rats produced a transient gain when only pilocarpine and acepromazine were administered to non-seized (normal) female rats. Weekly brief magnetic field exposures produced no statistically significant effect on the increasing obesity. A prenatal and postnatal environment that inhibits components of the NO pathway produced a mild reduction in the rate of weight gain. The results once again reiterate the complexity of the synergism between normal physiology, pharmaceutical treatments, and the changing electromagnetic environment within the twenty-first century [1].

\section{Acknowledgements}

We thank Dr. George A. Bubenik and Dr. Glenn Parker for their support. The corresponding author dedicates this document and research to the 20 undergraduate and graduate Behavioural Neuroscience students who fearlessly initiated litigation against a previous university administration who engaged in nefarious agreements with the Canadian Council on Animal Care to terminate these experiments and to compromise the reputations of the researchers involved with this area of investigation.

\section{References}

[1] Whissell, P.D. and Persinger, M.A. (2007) Emerging Synergisms between Drugs and Physiologically-Patterned Magnetic Fields: Implications for Neuorpharmacology and the Human Population in the Twenty-First Century. Current Neuropharmacology, 5, 278-288. http://dx.doi.org/10.2174/157015907782793603

[2] Thomas, J.R., Burch, L.S. and Yeandle, S.S. (1979) Microwave Radiation and Chlordiazepoxide: Synergistic Effects on Fixed-Internal Behaviour. Science, 204, 1357-1358. http://dx.doi.org/10.1126/science.424759

[3] Omura, Y. and Losco, M. (1993) Electromagnetic Fields in the Home Environment as Potential Contributing Factors for Induction of Oncogen C-Fos, Abl, Oncogen C-Fos Ab 2, Integrin Alpha 5, Beta 1, and Development of Cancer and Amino Acid composition of Food and Living Human Brain. International Journal of Acupuncture and Electro Therapeutics Research, 18, 33-73.

[4] Baluduzzo, M., Milone, F.F., Minenli, T.A., Pittaro-Cador, I. and Turicchia, L. (2011) Mathematical Phenomenology of Neural Stimulation by Periodic Fields. Nonlinear Dynamics Psychological Life Sciences, 69, 2765-2773.

[5] Persinger, M.A. and Belanger-Chellew, G. (1999) Facilitation of Seizures in Limbic Epileptic Rats by Complex 1 Microtesla Magnetic Fields. Perceptual and Motor Skills, 89, 486-492. http://dx.doi.org/10.2466/pms.1999.89.2.486

[6] St-Pierre, L.S. and Persinger, M.A. (2005) Extreme Obesity in One Year Old Female Rats in Which Seizures Were Induced before Puberty by Lithium/Pilocarpine Followed by a Single Injection of Acepromazine. Epilepsy and Behavior, 7, 346-347. http://dx.doi.org/10.1016/j.yebeh.2005.06.009

[7] Persinger, M.A., Bureau, Y.R.J., Kostakos, M., Peredery, O. and Falter, H. (1993) Behaviors of Rats with Insidious, Multifocal Brain Damage Induced by Seizures Following Single Peripheral Injections of Lithium and Pilocarpine. Physiology and Behavior, 53, 849-866. http://dx.doi.org/10.1016/0031-9384(93)90261-D

[8] Racine, R.J. (1972) Modification of Seizure Activity by Electrical Stimulation. II. Motor Seizures. Electroencephalography and Clinical Neurophysiology, 32, 281-294. http://dx.doi.org/10.1016/0013-4694(72)90177-0

[9] Persinger, M.A., O’Connor, R.P., Bureau, Y.R.J., Parker, G.H., Peredery, O. and Zegil, M. (2001) Synergistic Induction of Severe Hypothermia (Pokilothermia) by Limbic Seizures, Acepromazine and Physical Restraint: Role of Noradrenergic Alpha-1 Receptors. Pharmacology, Biochemistry and Behavior, 70, 341-352.

http://dx.doi.org/10.1016/S0091-3057(01)00619-0

[10] Loscher, W., Brandt, C. and Ebert, U. (2003) Excessive Weight Gain in Rats over Extended Kindling of the Baso- 
lateral Amygdala. Neuropharmacology and Neurotoxicology, 14, 1829-1832.

[11] King, B.M., Cook, J.T., Rossiter, K.N. and Rollins, B.L. (2003) Obesity-Inducing Amygdale Lesions: Examination of Anterograde Degeneration and Retrograde Transport. American Journal of Regulation and Integrated Comparative Physiology, 284, R965-R982.

[12] St-Pierre, L.S., Parker, G.H., Bubenik, G.A. and Persinger, M.A. (2007) Enhanced Mortality of Rat Pups Following Inductions of Epileptic seizures after Perinatal Exposures to 5 nT, 7 Hz Magnetic fields. Life Sciences, 81, 1496-1500. http://dx.doi.org/10.1016/j.lfs.2007.09.013

[13] Francis, D., Diorio, J., Liu, D. and Meany, M.J. (1999) Nongenomic Transmission across Generations of Maternal Behaviour and Stress Responses in the Rat. Science, 286, 1155-1158. http://dx.doi.org/10.1126/science.286.5442.1155

[14] Lado, W.E. and Persinger, M.A. (2012) Spatial Memory Deficits and Their Correlations with Clusters of Shrunken Soma in Cortices and Limbic System Following a "Mild" Mechanical Impact to the Dorsal Skull in Female Rats. Journal of Behavioral and Brain Sciences, 2, 333-342. http://dx.doi.org/10.4236/jbbs.2012.23038

[15] Martin, L.J., Koren, S.A. and Persinger, M.A. (2004) Thermal Analgesic Effects from Weak, Complex Magnetic Fields and Pharmacological Interactions. Pharmacology, Biochemistry and Behavior, 78, 217-277. http://dx.doi.org/10.1016/j.pbb.2004.03.016

[16] Thomas, A.W., Kavaliers, M., Prato, F.S. and Ossenkopp, K.P. (2002) Antinociceptive Effects of a Pulsed Magnetic Field in the Land Snail: Cepaea nemoralis. Neuroscience Letters, 222, 107-110. http://dx.doi.org/10.1016/S0304-3940(97)13359-6

[17] Thomas, A.W., Kavaliers, M., Prato, F.S. and Ossenkopp, K.P. (1997) Pulsed Magnetic Field Induced “Analgesia” in the Land Snail, Cepaea normalis, and the Effects of $M u$, Delta, and Kappa Opioid Receptor Agonists/Antagonists. Peptides, 18, 703-709.

[18] Whissell, P.D. and Persinger, M.A. (2007) Developmental Effects of Prenatal Exposure to Extremely Weak 7 Hz Magnetic Fields and Postnatal Nitric Oxide Modulation in the Wistar Albino Rat. International Journal of Developmental Neuroscience, 25, 433-439. http://dx.doi.org/10.1016/j.ijdevneu.2007.09.001

[19] Su, H., Sochivko, D., Becker, A., Chen, J., Jiang, Y., Yaari, Y. and Beck, H. (2002) Upregulation of T-Type Ca ${ }^{2+}$ Channels Causes a Long-Lasting Modification of Neuronal Firing Mode after Status Epilepticus. Journal of Neuroscience, 22, 3645-3655.

[20] McKay, B.E., Persinger, M.A. and Koren, S.A. (2000) Exposure to a Theta-Burst Patterned Magnetic Field Impairs Acquisition and Consolidation for Contextual but Not Conditioned Fear in Rats. Neuroscience Letters, 292, 99-102. http://dx.doi.org/10.1016/S0304-3940(00)01437-3

[21] St-Pierre, L.S., Mazzuchin, A. and Persinger, M.A. (2008) Altered Blood Chemistry and Hippocampal Morphology in Adult Rats Following Prenatal Exposure to Physiologically-Patterned, Weak (50 to 500 Nano Tesla Range) Magnetic Fields. International Journal of Radiation Biology, 84, 325-335. http://dx.doi.org/10.1080/09553000801953300

[22] Buckner, C.A., Buckner, A.L., St-Pierre, L.S., Koren, S.A., Persinger, M.A. and Lafrenie, R.M. (2014) Inhibition of B16-BL6 Cell Proliferation Using a Weak Frequency-Modulated Electromagnetic Field Pattern. In Submission.

[23] Saroka, K.S. and Persinger, M.A. (2013) Potential Production of Hughling Jackson's "Parasitic Consciousness” by Physiologically-Patterned Weak Transcerebral Magnetic Fields: QEEG and Source Localization. Epilepsy and Behavior, 28, 395-407. http://dx.doi.org/10.1016/j.yebeh.2013.05.023

[24] Adey, W.R. (1980) Frequency and Power Windowing in Tissue Interactions with Weak Electromagnetic Fields. Proceedings of International Electrical Engineering, 68, 119-125. http://dx.doi.org/10.1109/PROC.1980.11591

[25] Phan, T.D., Kistler, L., Klecker, B., Harerendel, G. and Paschmann, G. (2000) Extended Magnetic Connection at the Earth's Magnetopause from Direction of Bi-Directional Jets. Science, 404, 848-850.

[26] Galley, P.C. (1999) Membrane Potential and Time Requirements for Detection of Weak Signals by Voltage-Gated Ion Channels. Bioelectromagnetics, 20, 102-109.

[27] Ahmed, Z. and Wierasko, A. (2008) The Mechanism of Magnetic Field-Induced Increase of Excitability in Hippocampal Neurons. Brain Research, 1221, 30-40. http://dx.doi.org/10.1016/j.brainres.2008.05.007

[28] Williams, T.L. (1992) Phase Coupling by Synaptic Spread in Chains of Coupled Neuronal Oscillators. Science, 258, 662-664. http://dx.doi.org/10.1126/science.1411575

[29] Persinger, M.A. (2010) 10-20 Joules as a Neuromolecular Quantum in Medicinal Chemistry: An Alternative Approach to Myriad Molecular Pathways. Current Medicinal Chemistry, 17, 3094-3098. http://dx.doi.org/10.2174/092986710791959701

[30] Persinger, M.A. and Koren, S.A. (2007) A Theory of Neurophysics and Quantum Neuroscience: Implications for Brain Function and the Limits of Consciousness. International Journal of Neuroscience, 117, 157-175. http://dx.doi.org/10.1080/00207450500535784 
[31] Calvo, A.C. and Azana, M.J. (1999) Synaptic Neurone Activity under Applied 50 Hz Alternating Magnetic Fields. Comparative Biochemistry and Physiology Part C, 124, 99-107.

[32] Ishido, M., Nitta, H. and Kubuto, M. (2001) Magnetic Fields of $50 \mathrm{~Hz}$ at 1.2 MicroT as Well as 100 MicroT Cause Uncoupling of Inhibitory Pathways of Adenylyl Cyclase Mediated by Melatonin 1a Receptor in MF-Sensitive MCF-7 Cells. Carcinogenesis, 22, 1043-1048. http://dx.doi.org/10.1093/carcin/22.7.1043

[33] Sakper, S.D., Ancona, B., Facci, L., Franceschini, D. and Giusti, P. (1998) Melatonin Prevents the Delayed Death of Hippocampal Neurons Induced by Enhanced Excitatory Neurotransmission and the Nitridergic Pathway. The FASEB Journal, 12, 725-731.

[34] Bubenik, G.A. (2002) Review: Gastrointestinal Melatonin: Localization, Function and Clinical Relevance. Digestive Disease and Science, 47, 2336-2348. http://dx.doi.org/10.1023/A:1020107915919

[35] Bubenik, G.A., Blask, D.E., Brown, G.M., Maestroni, G.J.M., Pang, S.F., Reiter, R.J., Viswanathan, M. and Ziaspel, N. (1998) Prospects of Clinical Utilization of Melatonin. Biological Signals and Receptors, 7, 195-219. http://dx.doi.org/10.1159/000014545

[36] St-Pierre, L.S., Persinger, M.A. and Koren, S.A. (1998) Experimental Induction of Intermale Aggressive Behaviour in Limbic Epileptic Rats by Weak, Complex Magnetic Fields: Implications for Geomagnetic Activity and the Modern Habitat? International Journal of Neuroscience, 96, 149-159. http://dx.doi.org/10.3109/00207459808986464

[37] Stewart, L.S. and Persinger, M.A. (2000) Pretraining Exposure to Physiologically Patterned Electromagnetic Stimulation Attenuates Fear-Induced Analgesia. International Journal of Neuroscience, 100, 91-98. http://dx.doi.org/10.3109/00207450008999679

[38] Shimuzi, H. and Mori, M. (2005) The Brain-Adipose Axis: A Review of Involvement of Molecules. Nutritional Neuroscience, 8, 7-20. http://dx.doi.org/10.1080/10284150500047245

[39] Ahima, R.S. (2005) Central Actions of Adipocyte Hormones. Trends in Endocrinology and Metabolism, 16, 307-313. http://dx.doi.org/10.1016/j.tem.2005.07.010

[40] Peredery, O., Persinger, M.A., Parker, G. and Mastrosov, L. (2000) Temporal Changes in Neuronal Dropout Following Inductions of Lithium/Pilocarpine Seizures in the Rat. Brain Research, 881, 9-17. http://dx.doi.org/10.1016/S0006-8993(00)02730-X

[41] Bureau, Y.R.J., Persinger, O. and Persinger, M.A. (1994) Concordance of Quantitative Damage within the Diencephalon and Telencephalon Following Systemic Pilocarpine (380 mg/kg) or Lithium (3 mEq/kg)/Pilocarpine (30 mg/kg) Induced Seizures. Brain Research, 648, 265-269. http://dx.doi.org/10.1016/0006-8993(94)91126-6

[42] Fraser-Smith, A.C. (1992) The Natural Background Levels of 50/60 Hz Radio Noise. IEEE Transactions on Electromagnetic Compatibility, 34, 330-337. http://dx.doi.org/10.1109/15.155849

[43] Dopson, J., St-Pierre, T., Wieser, H.G. and Fuller, M. (2000) Changes in Paroxysmal Brainwave Patterns of Epileptics by Weak-Magnetic Field Stimulation. Bioelectromagnetics, 21, 94-95.

[44] Marquez, A.V., Farias, S.T., Apperson, M., Koopmans, S., Jorgensen, J., Shatzel, A. and Alsaadi, T.M. (2004) Psychogenic Nonepileptic Seizures Are Associated with Increased Risk of Obesity. Epilepsy and Behavior, 5, 88-93. http://dx.doi.org/10.1016/j.yebeh.2003.10.019

[45] Bhatt, R., Bhatt, S., Rameswhar, P. and Siegel, A. (2004) Amygdaloid Kindled Seizures Induce Weight Gain and Reflects the Left Hemispheric Dominance in Rats. Physiology and Behavior, 82, 581-587. http://dx.doi.org/10.1016/j.physbeh.2004.05.005

[46] Persinger, M.A. and Dupont, M.J. (2004) Emergence of Spontaneous Seizures during the Year Following Lithium/Pilocarpine-Induced Epilepsy and Neuronal Loss within the Right Temporal Cortices. Epilepsy and Behavior, 5, 440-445. http://dx.doi.org/10.1016/j.yebeh.2004.03.007

[47] Smith, S.L., Harrold, J.A. and Williams, G. (2002) Diet-Induced Obesity Increases Mu Opioid Receptor Binding in Specific Regions of the Rat Brain. Brain Research, 953, 215-222. http://dx.doi.org/10.1016/S0006-8993(02)03291-2

[48] Hogan, M.V. and Wieraszko, A. (2004) An Increase in cAMP Concentration in Mouse Hippocampal Slices Exposed to Low-Frequency and Pulsed Magnetic Fields. Neuroscience Letters, 366, 43-47. http://dx.doi.org/10.1016/j.neulet.2004.05.006

[49] Nie, K. and Henderson, A. (2003) MAP Kinase Activation of Cells Exposed to a $60 \mathrm{~Hz}$ Electromagnetic Field. Journal of Cell Biochemistry, 90, 1197-1206. http://dx.doi.org/10.1002/jcb.10704

[50] Peredery, O., Persinger, M.A., Blomme, C. and Parker, G. (1992) Absence of Maternal Behaviour in Rats with Lithium/Pilocarpine Seizure-Induced Brain Damage: Support of MacLean’s Triune Brain Theory. Physiology and Behavior, 52, 665-661. http://dx.doi.org/10.1016/0031-9384(92)90395-I

[51] Dupont, M.J., McKay, B.E., Parker, G. and Persinger, M.A. (2004) Geophysical Variables and Behaviour. XCIX. Reductions in Numbers of Neurons within the Perisolitary Nucleus in Rats Exposed Perinatally to a Magnetic Pattern De- 
signed to Imitate Geomagnetic Pulsations: Implications for Sudden Infant Death. Perceptual and Motor Skills, 98, 958966. http://dx.doi.org/10.2466/pms.98.3.958-966

[52] Giruado, S.Q., Kotz, C.M., Billington, C.J. and Levine, A.S. (1998) Association between the Amygdale and Then Nucleus of the Solitary Tract in Mu-Opoid Induced Feeding Behaviour. Brain Research, 802, 184-188. http://dx.doi.org/10.1016/S0006-8993(98)00602-7

[53] Persinger, M.A. and Koren, S.A. (2001) Experiences of "Spiritual Visitation and Impregnation": Potential Induction by Frequency-Modulated Transients from an Adjacent Clock. Perceptual and Motor Skills, 92, 35-36. http://dx.doi.org/10.2466/pms.2001.92.1.35 
Scientific Research Publishing (SCIRP) is one of the largest Open Access journal publishers. It is currently publishing more than 200 open access, online, peer-reviewed journals covering a wide range of academic disciplines. SCIRP serves the worldwide academic communities and contributes to the progress and application of science with its publication.

Other selected journals from SCIRP are listed as below. Submit your manuscript to us via either submit@scirp.org or Online Submission Portal.
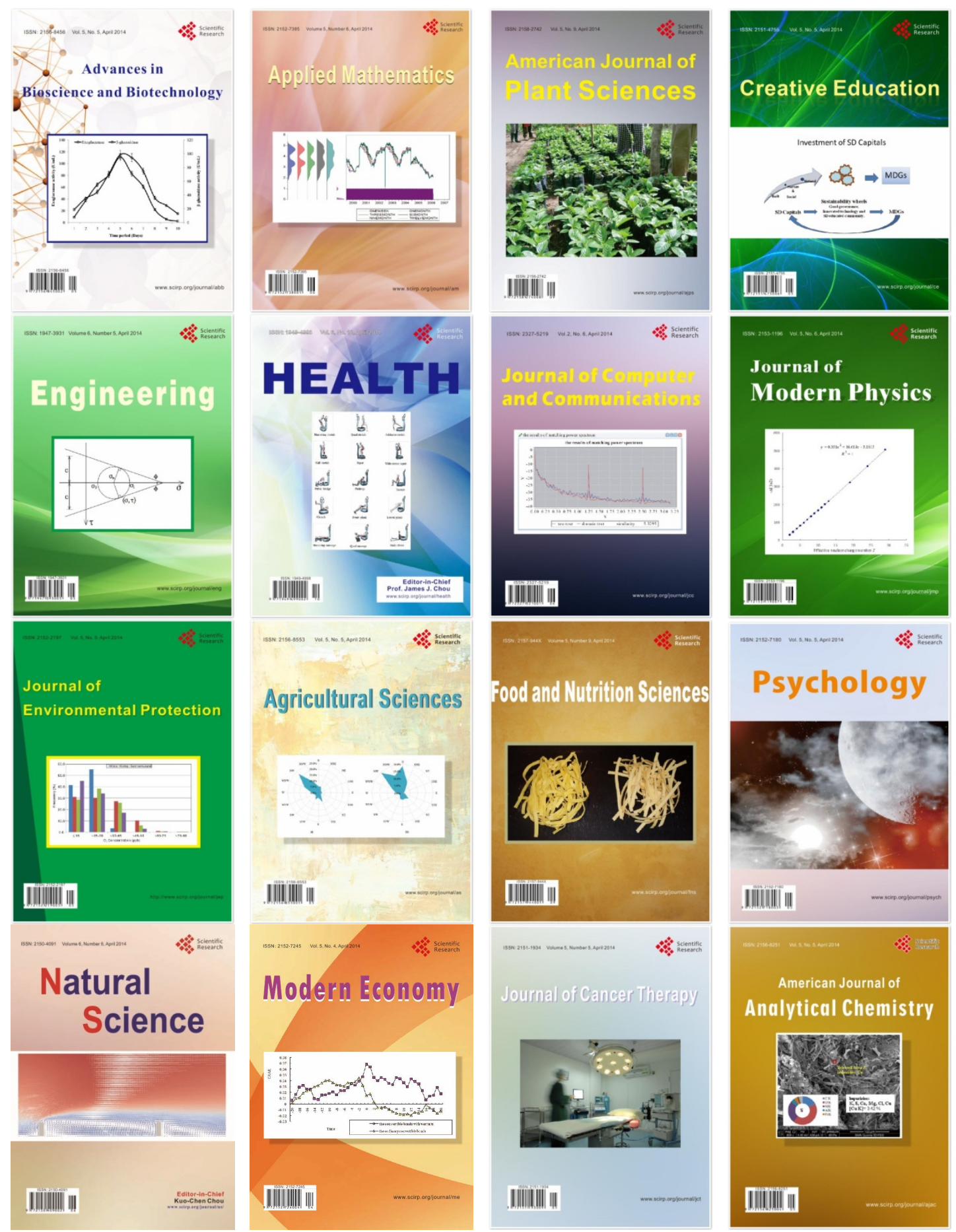